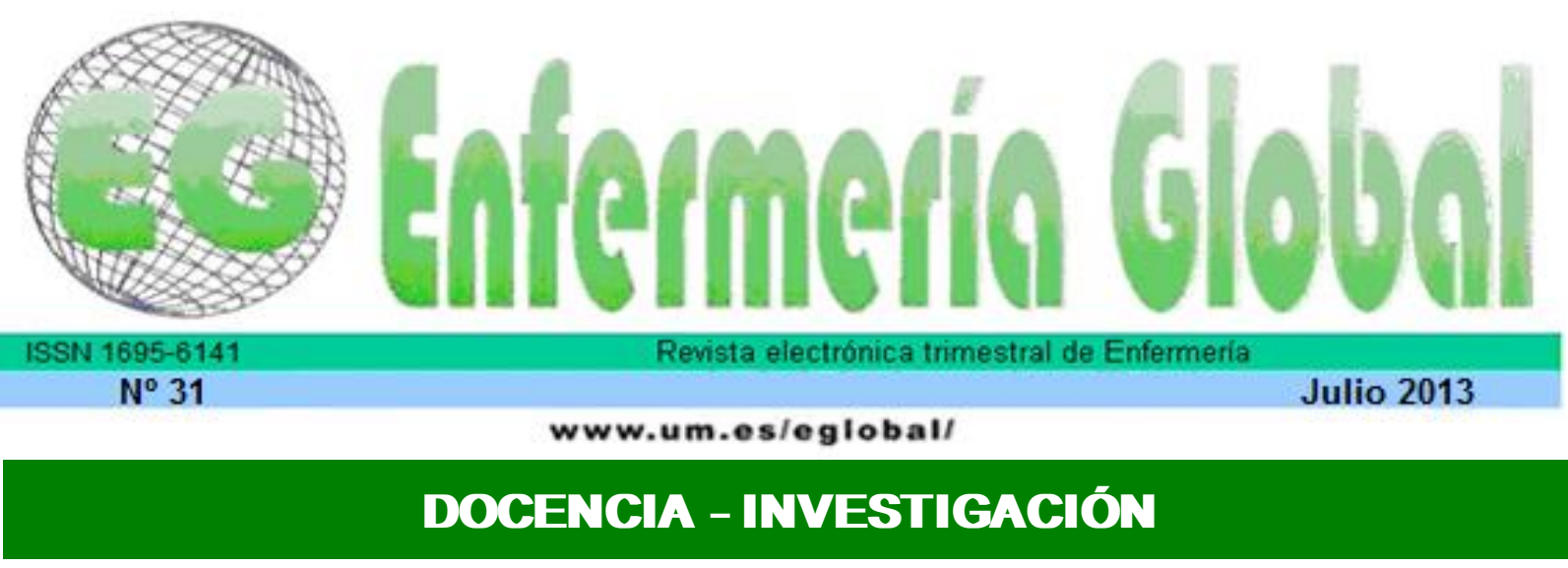

\title{
Trabajadores de la salud y sus significados en torno a la adherencia al tratamiento de la tuberculosis
}

Health workers and their meanings towards the adhesion to the tuberculosis treatment

\begin{abstract}
${ }^{*}$ Muñoz Sánchez, Alba Idaly ${ }^{* *}$ Cruz Martínez, Óscar Andrés ${ }^{* * *}$ Rubiano Mesa, Yurian Lida

*Doctora en Enfermería. Docente Facultad de Enfermería. E-mail: albaidalymunoz@gmail.com **Enfermero *** Enfermera. Magister en Salud Familiar. Docente Facultad de Enfermería. Universidad Nacional de Colombia.
\end{abstract}

Palabras clave: tuberculosis; paciente ambulatorio; cumplimiento de la medicación; investigación en los servicios de salud; trabajadores de la salud

Keywords: tuberculosis; outpatient; medication fulfillment: research of the health services; health workers.

\section{RESUMEN}

Objetivos: Se propuso identificar el concepto de adherencia al tratamiento de la tuberculosis, las causas que la limitan o que la fortalecen, a través del análisis de los significados de los trabajadores de la salud en una localidad de Bogotá.

Métodos: Estudio cualitativo, alcance descriptivo. Perspectiva teórica metodológica, hermenéuticadialéctica. Se entrevistaron 18 trabajadores de la salud. La recolección de los datos se realizó en el año 2010.

Resultados: Los participantes conciben la adherencia al tratamiento como un fenómeno complejo: conocimiento sobre la importancia del tratamiento, voluntad del mismo para la toma del tratamiento y la asistencia a controles, además integra factores programáticos, como las visitas del equipo de salud, la flexibilidad en la entrega de los medicamentos, la construcción y consolidación de redes de apoyo para los portadores, el apoyo de la familia, entre otros.

Factores que limitan la adherencia: Se reconoce el proceso salud-enfermedad-tuberculosis como categoría que interfiere en el fenómeno adherencia, este integra el estigma social, evidenciado en estereotipos y prejuicios que relacionan la enfermedad. El sistema de salud es visto como 
fragmentado. El desconocimiento de la enfermedad, en pacientes y trabajadores, genera temor durante el proceso de atención.

Aspectos que fortalecen la adherencia: Conocimiento sobre la enfermedad e importancia del tratamiento. Las acciones intersectoriales e interinstitucionales, las redes familiares, la atención humanizada y con calidad de los profesionales de salud, y el contar con un sistema de seguimiento y comunicación permanente.

Conclusiones: Se reitera la importancia de estudios para la comprensión y el estudio del proceso adherencia a la tuberculosis. En este sentido, se deben desarrollar múltiples estudios, no solo diagnósticos, sino de intervenciones desde diversas ópticas (academia, población civil, instituciones de salud y entes gubernamentales, entre otros actores).

\section{ABSTRACT}

Objectives: To identify the concept of adhesion to the treatment of tuberculosis, the causes that restrict or strength it, by means of the analysis of the meanings of the health workers in a locality in Bogota.

Methods: Qualitative, descriptive study. Methodological theory, hermeneutics-dialectics approach. 18 health workers were interviewed. The recording of the data was done in 2010.

Results: Participants conceive an adhesion to the treatment as a complex phenomenon: the knowledge about the importance of the treatment, the person's own will to take the treatment and to attend revisions. Moreover, it integrates programmatical factors, such as the visits to the health team, the flexibility regarding the delivery of medicines, the construction and consolidation of support nets for the carriers, the family support, among others.

Factors that restrict the adherence: The process health-disease-tuberculosis is recognized as a category that interferes in the adherence phenomenon; this integrates the social stigma, evidenced in stereotypes and prejudices that relate the disease. The health system is seen as broken. The ignorance of the disease, in both patients and workers, generates a fear during the process of attention.

Aspects that strength the adherence: Knowledge of the disease and importance of the treatment. The inter-sectorial and inter-institutional actions, the family nets, the human and quality attention of the health professionals, and taking into account with a system of permanent tracking and communication are the main ones.

Conclusions: The importance of studies for the understanding and the study of the process of adherence is reiterated. In this sense, several studies must be developed, not only diagnosis studies, but also interventions from diverse viewpoints (academy, civil population, health institutions and governmental beings, among others).

\section{INTRODUCCIÓN}

La infección por Mycobacterium tuberculosis en el mundo afecta al 95\% de los países en vía de desarrollo ${ }^{1}$. Si bien los avances tecnológicos en la biomedicina, el mejoramiento de las condiciones de vida y las estrategias de tratamiento se constituyen en logros de gran importancia para el control de la tuberculosis, no han sido suficientes para que la enfermedad desaparezca del mundo; Últimas cifras disponibles del Reporte Global de Tuberculosis emitido por la Organización Mundial de la Salud (OMS), estimó que para el año 2010 murieron a causa de Tuberculosis 
1,1 millones de adultos. La enfermedad presentó una incidencia de 8,8 millones, una prevalencia de 12 millones, un total de 1,2 millones de casos de co-infección de pacientes con Tuberculosis asociada al Virus de Inmunodeficiencia Humana o (TBVIH/SIDA) y 0.65 millones de casos de Multidrogoresistencia o (MDR-TB), a los medicamentos efectivos que curan la enfermedad. ${ }^{1}$

En Colombia, según reporte de la OMS al año 2010, el país registró una incidencia aproximadamente de 11.420 casos, una mortalidad total de aproximadamente 1.300 casos, asi como un porcentaje de $1.5 \%$ de casos de tuberculosis MDR- TB ${ }^{1}$. De igual forma, se ha registrado una detección de pacientes Sintomáticos Respiratorios para Tuberculosis en el año 2008 del 54\%, y se presentó un porcentaje de abandono de los pacientes al tratamiento del $9,2 \%{ }^{2}$.

En Bogotá, de acuerdo con los estudios realizados por la Secretaría Distrital de Salud y el análisis de los indicadores de la operación del programa de control de la tuberculosis, se identifica como uno de los principales problemas la baja captación de sintomáticos respiratorios por parte del personal de salud, ya que en la mayoría de las instituciones solo se capta entre el 1 y el 3\%, y la meta definida para el país es del $10 \%$. Además, las altas tasas de abandono, que alcanzan el $13 \%$, son uno de los indicadores de no adherencia al tratamiento en Bogotá, situación que agrava la farmacoresistencia a los fármacos y aumenta el riesgo de transmisión de la enfermedad $^{3}$.

Por su parte, el abandono de los pacientes a la toma del tratamiento se asocia a múltiples dimensiones, lo que hace que el análisis de adherencia sea complejo. En la adherencia confluyen diversos factores, que se derivan de la persona misma y su entorno (sociocultural, familiar, económico), de los factores de su enfermedad (esquema de tratamientos, coinfección, resistencia, etc.), de los factores relacionados con la prestación de servicios de salud (la efectividad de los programas para el control, en la búsqueda de sintomáticos respiratorios, diagnóstico oportuno, tratamiento, etc.) y en la relación que se establece entre los trabajadores de la salud y pacientes (buen trato, flexibilidad, comunicación, los conocimientos y creencias), en torno a la enfermedad ${ }^{4}$.

Existen estudios sobre adherencia al tratamiento de la tuberculosis desde la perspectiva de los pacientes y los colectivos a través de metodologías cuantitativas ${ }^{5,6}$, 7,8 , pero son escasos a nivel de América Latina, y específicamente en Colombia, y más escasos aún son los de corte cualitativo con trabajadores de la salud, quienes juegan un papel fundamental en la atención directa y orientación a los pacientes y comunidades sobre la enfermedad. Así, se propuso identificar las causas que limitan o fortalecen la adherencia al tratamiento de la tuberculosis, a través del análisis de los significados de los trabajadores de la salud de una localidad de Bogotá. Esta investigación fue financiada por la Dirección de Investigación de la Universidad Nacional de Colombia-Sede Bogotá.

Estudios han evidenciado que los conocimientos de los trabajadores de la salud son factores claves para la captación de casos y la adherencia al tratamiento ${ }^{4}$, y que los significados que los trabajadores de la salud poseen sobre la enfermedad pueden contribuir o no en la adherencia al tratamiento ${ }^{7}$.

Por lo expuesto se justifica la necesidad de identificar los aspectos que contribuyen o limitan la adherencia al tratamiento de la enfermedad, a través del análisis de los 
significados de trabajadores de la salud y de los portadores de la enfermedad en la localidad de Bogotá. Este estudio pretende revelar información que permita direccionar acciones futuras desde las tres misiones de la academia: docencia, extensión e investigación, teniendo en cuenta la voz de las personas que realizan la atención en salud a los pacientes diagnosticados con tuberculosis del programa de control del Distrito Capital.

\section{OBJETIVO GENERAL}

Identificar los aspectos que contribuyen o limitan la adherencia al tratamiento de la tuberculosis, a través del análisis de los significados de trabajadores de la salud de una localidad de Bogotá.

\section{METODOLOGÍA}

\section{Referencial teórico:}

El concepto de hermenéutica ha ganado connotaciones diferentes en el contexto de la tradición científica de la época moderna. Gadamer, apunta que a través de la hermenéutica filosófica se alcanza la comprensión, siempre y cuando el sujeto investigador exponga en el proceso todos sus presupuestos y se muestre que existe una tensión en la comprensión en los diferentes momentos históricos, la cultura, las clases sociales, la vida; esto le da vida y tensión a la comprensión, que tiene un carácter supraobjetivo; así el texto y el intérprete tienen sus propios horizontes ${ }^{5}$

Apunta Grondin que el único principio metodológico de la hermenéutica de Gadamer "es que quizás sea el otro el que pudiera tener la razón" ${ }^{6}$, así que califica el escuchar como un arte y destaca la importancia de reconocer al otro. La hermenéutica es definida por Gadamer "como una praxis, el arte de comprender y hacer comprender [...], hay que ejercitar sobre todo el oído, la sensibilidad para las predefiniciones, los preconceptos y presignificaciones que subyacen de los conceptos" 7. El arte del que trata Gadamer "es el del anuncio, la traducción, la explicación y la interpretación, e incluye, obviamente, el arte de la comprensión que subyace y que se requiere cuando no está claro y no es inequívoco el sentido de algo" 7 .

Así mismo, Gadamer define la dialéctica como "el arte de llevar una conversación consigo mismo y de perseguir el entendimiento consigo mismo. Es el arte de pensar, pero equivale al arte de indagar el significado de lo que se piensa y se dice" ${ }^{8}$. Estos dos conceptos exponen la importancia del diálogo en la investigación con esta perspectiva y reconocen el valor inestimable del encuentro entre investigador y sujeto de investigación, como un proceso en constante transformación, dinámico, en movimiento, que es propio en el método cualitativo.

Las ciencias sociales, desde su génesis en el siglo XVIII y su consolidación en el siglo XIX, afirman que el análisis socio-histórico fundamenta su esencia, y que no se sujetan ni se aprehenden ni se miden con las metodologías y parámetros del conocimiento de las ciencias naturales. Los fenómenos históricos sociales, como el proceso saludenfermedad, no se pueden tomar como fenómenos concretos y generalizables ${ }^{8}$, dado que bajo el abordaje cualitativo cada objeto de investigación es único, el análisis del fenómeno es histórico e irrepetible; en otros términos, el objetivo de la investigación cualitativa no es revalidar o ampliar las experiencias generales para llegar al conocimiento de fenómenos, como los que se presentan en los servicios de salud, 
vivenciar procesos salud-enfermedad, evaluar los servicios de salud, analizar las condiciones de trabajo de las distintas categorías profesionales o los procesos de trabajo en sectores productivos, entre otros, sino comprender el proceso saludenfermedad, la manera como esos fenómenos se conciben, comprender que su significado es diferenciado en cada sujeto y lugar.

La articulación de la dialéctica y la hermenéutica permite abordar el análisis de la realidad, en este caso de los significados que trabajadores de la salud en los aspectos relacionados con la adherencia al tratamiento de la tuberculosis. La dependencia y complementariedad entre estos abordajes, permite comprender lo general y las particularidades de la realidad en un tiempo y espacio, teniendo en cuenta la historia de cada participante de esta investigación.

En el marco del presente estudio, el primer paso que se llevó a cabo fue el acercamiento para las autorizaciones respectivas con las instituciones, sus directivas y funcionaros. Como criterios de inclusión para los trabajadores de la salud se definió que fueran médicos, enfermeras y auxiliares de enfermería u otros profesionales que prestaran atención directa a personas que padecen tuberculosis, que llevaran un año de experiencia en esta área y que aceptaran voluntariamente participar en el estudio. El número de trabajadores para el estudio no se estableció con anticipación, en virtud de la metodología adoptada; el estudio se desarrolló en una localidad de la ciudad de Bogotá, en una Empresa Social del Estado y en su red de Unidades de Salud Primarias públicas y privadas adscritas al programa de prevención y control de la tuberculosis y al sistema de notificación de la Secretaria Distrital de Salud. El estudio se puso a consideración del Comité de Ética de la Facultad de Enfermería de la Universidad Nacional de Colombia, el cual le dio su aprobación. Los datos se recogieron en las fechas y en un lugar establecido con anterioridad de común acuerdo con el personal de la institución. Se dio a conocer a los sujetos el objeto de estudio y se obtuvo el consentimiento informado

El límite de participantes fue identificado en la medida en que se repetían los temas en la información recogida por medio de las entrevistas semiestructuradas. Para el presente estudio se obtuvo la saturación de datos con 18 entrevistas a trabajadores de la salud. Para la recolección de los datos se utilizó una entrevista siguiendo un formato semiestructurado, con una duración aproximada de una hora con cada uno de los participantes. Dada la perspectiva en la cual se inscribe esta investigación, el análisis fue un proceso interpretativo que permitió la compresión de los significados de los trabajadores sobre la "adherencia al tratamiento de la tuberculosis". Este proceso se inicio desde el momento de la recolección de la información y se extendió aun después de terminarla; consistió en la codificación, clasificación, comparación y contrastación de los datos, para organizarlos en familias y categorías que surgieron de los mismos datos y de la reflexión teórica.

Para lo anterior, se contó con el apoyo del programa Atlas.ti. Simultáneamente se fueron encontrando relaciones entre las familias y categorías construyendo, a su vez, categorías mayores que contenían a las otras. Lo anterior permitió la lectura del fenómeno de la adherencia al tratamiento antituberculoso por parte de los participantes como un "todo" que dio sentido a las partes. Los resultados se validaron y se dieron a conocer a cada uno de los participantes de manera individual, con el objetivo de propiciar la reflexión en torno a ellos y a sus propias vivencias, y así escuchar atentamente sus nuevas inquietudes, comentarios y críticas. Las entrevistas fueron grabadas y transcritas en su totalidad por los investigadores, y fueron 
analizadas buscando la racionalidad y su sentido, con foco en los significados relacionados con la adherencia al tratamiento de la tuberculosis de los trabajadores de la salud. Los resultados finales fueron presentados a todos los trabajadores de las diversas localidades de salud de Bogotá.

\section{RESULTADOS}

\section{Caracterización de los trabajadores de la salud}

Se logró contar con la participación de 18 trabajadores de la salud de la localidad: 15 mujeres y 3 hombres, con un promedio de edad de 34 años. Según tipo de institución, se conformó por nueve del sector público y nueve del sector privado. Según el cargo, los entrevistados fueron nueve enfermeras, cuatro médicos, dos auxiliares de enfermería, una bacterióloga, una odontóloga y una psicóloga.

Se identificó una gran categoría, denominadas (Adherencia al tratamiento de la tuberculosis). Las subcategorias aspectos individuales y sociales-programáticos. Este proceso también se apoyó con el uso del Sofware Atlas Tic Dichas categorías corresponden a elementos estructurales de donde se integran los aspectos que fortalecen la adherencia, los aspectos que la limitan.

\section{Construcción del concepto de Adherencia al tratamiento de la tuberculosis}

La adherencia en el tratamiento de la tuberculosis es uno de los elementos claves para que se dé la curación de los portadores. La adherencia es concebida como un fenómeno complejo, desde la perspectiva de los trabajadores de la salud. En este sentido, adherencia significa el cumplimiento y la asistencia permanente del paciente a la toma del medicamento. Los significados de adherencia se representan desde lo individual, lo social y lo programático, tal como se representa en el gráfico $\mathrm{N}$. $^{\circ}$.

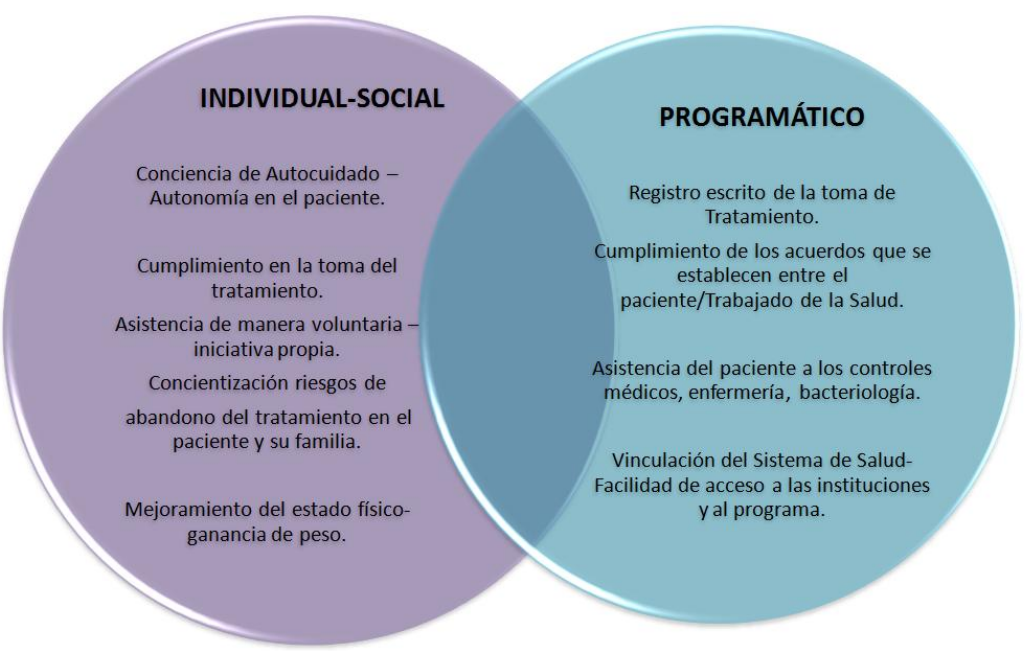

CONCEPTO DE ADHERENCIA AL TRATAMIENTO

Gráfico N. ํ. Significados de la adherencia 


\section{- Adherencia: individual - social}

La adherencia en la perspectiva de los trabajadores se relaciona, de forma general, con los conocimientos y la comprensión que tienen los pacientes con su situación de salud-enfermedad.

Un trabajador de la salud menciona: P10: (10:43) "Yo creo que la adherencia es, primero, conciencia de qué es la salud como tal, personal, autocuidado". Otro trabajador de la salud expresa lo que es la adherencia: P11 (11:73) "Un paciente que no haya que estarle recordando que necesita tomarse su tratamiento para recuperarse. ¡Eso es adherencia al tratamiento!”.

La adherencia, como aspecto fundamental, se define por el cumplimiento de la persona en la toma de los medicamentos: P8: (8:5) "Adherencia es que el mismo usuario reconozca que debe tomarse el tratamiento, que debe ir a tomárselo"; (12:36) "Adherencia es un paciente que conoce su enfermedad, que no se queda solo con la información que le damos acá, sino que averigua”. Otro trabajador de la salud define adherencia como: P1: (1:13) "El paciente que llegó con condiciones de salud muy malas, nutricionalmente también decaído y va viendo cómo va mejorando su estado, el peso, todo".

Como fenómeno multidimensional, la adherencia es referida por los trabajadores en los siguientes términos: P15: (15:25) "Es tener conciencia de qué riesgos le pueden dar a él y a la familia y que cumpla el tratamiento". La adherencia al tratamiento implica para el paciente la conciencia de tratar la enfermedad, pero también involucra la identificación de los riesgos que puede traer consigo su incumplimiento o abandono del tratamiento.

Finalmente, la adherencia se reafirma en el discurso del trabajador de la salud como la voluntad y el cumplimiento permanente y constante del paciente: P18: (18:42) "La persona que conscientemente, que por su propia voluntad va, asiste a los controles médicos de bacteriología, medicina y enfermería"; P2: (2:18) "Aquel paciente que asiste puntualmente y que está al tanto de su salud".

\section{Adherencia: aspectos programáticos}

Desde esta categoría, los trabajadores de la salud relacionan la adherencia con procesos establecidos en el programa de tuberculosis y que se espera sean cumplidos por el paciente. Así afirma un trabajador: P7: (7:12) "De acuerdo a los controles que llevamos en la tarjeta"; P15: (15:25) "Que el paciente venga a sus citas, que sea juicioso con sus controles, a sus reuniones, que se haga sus baciloscopias".

La adherencia puede ser definida como la constancia escrita de la toma de los medicamentos, verificable en la ficha de tratamiento. También la adherencia se define por la asistencia de los pacientes a las actividades diagnósticas y terapéuticas que se establecen en el programa.

Finalmente, desde los aspectos programáticos se identifica que el acceso que tiene el paciente a las instituciones de salud están también implicados en la adherencia: P8: (8:5) "La adherencia está en el paciente y en la institución, dándole las facilidades para que tome el tratamiento", incluye la facilidad de acceso que se da en los horarios, la red de atención y la disponibilidad del tratamiento, entre otros. 
Teniendo en cuenta la superfamilia adherencia, y acorde con los objetivos planteados en la presente investigación, a continuación se describen tanto los factores que limitan como los que fortalecen esa adherencia al tratamiento de la tuberculosis, desde la perspectiva de los trabajadores de la salud en el escenario de estudio.

\section{Factores que limitan la adherencia al tratamiento}

Dentro de los factores que se identifican como limitantes en la adherencia al tratamiento de la tuberculosis en los pacientes desde la perspectiva de los trabajadores de la salud se observan, desde lo individual, aspectos socioeconómicos, fisiopatológicos, programáticos y la relación intersubjetiva entre el trabajador de la salud y el paciente, tal como se representa a continuación:

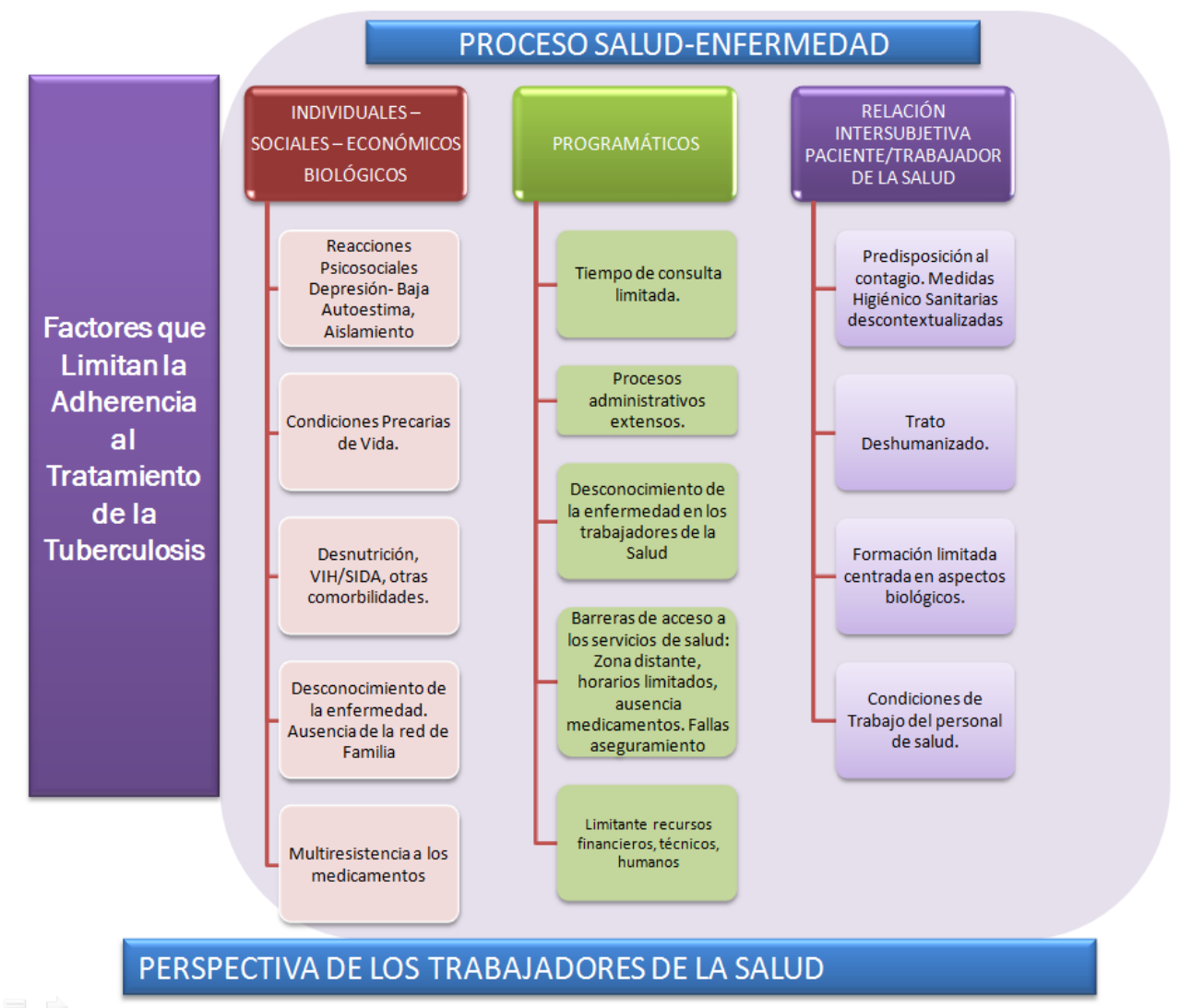

Gráfico N. ${ }^{\circ}$ 2. Factores que limitan la adherencia al tratamiento de la tuberculosis en la localidad, Bogotá, 2010

\section{- Aspectos individuales-socioeconómicos que limitan la adherencia}

Desde la perspectiva de los trabajadores de la salud, se identifican como limitantes para la consecución de la adherencia al tratamiento el estigma que acompaña la enfermedad, el aislamiento y el rechazo presentes en la construcción histórica y social del proceso salud-enfermedad-tuberculosis. También se identifican como limitantes los procesos fisiopatológicos, la coinfección VIH/SIDA, las precarias condiciones de vida, las creencias culturales, las fallas programáticas y las limitantes estructurales en la organización del sistema de salud. Así lo afirma un trabajador: P11: (11:8) "Ellos empiezan a presentar una baja autoestima, muy baja autoestima, pueden iniciar como un episodio depresivo en su vida por las múltiples barreras que tienen". 
La afectación de la enfermedad también involucra la dimensión social; así lo refiere el siguiente texto: P11: (1:8) "Bien sea para conseguir hasta un trabajo, como el rechazo que se inicia en las familias por las creencias que presentan"; (P12: 11:8) "Ellos mismos, por sus propias creencias, empiezan a aislarse de la sociedad, y la gente, al ver el aislamiento, piensa de verdad que es algo que no tiene cura". Estos sentimientos y creencias afectan el cotidiano de los pacientes en su relación con su familia, su círculo social, se afecta su trabajo y también su situación socioeconómica; P11: (11:7) "El hecho de que la persona no tenga una red de apoyo estable, una familia que la apoye, un amigo, y esté sola, eso la hace mucho más vulnerables".

Por otro lado, se identifican en las entrevistas de los trabajadores de la salud aspectos relacionados con la situación socioeconómica de las personas, y cómo esta influye directamente en el acceso al tratamiento para el logro de la adherencia. En efecto, un trabajador manifiesta: P15: (15:19) “La parte económica puede limitar la adherencia, por el desplazamiento a los centros de salud, para que ellos se adhieran al tratamiento".

La estrategia de tratamiento supervisado, implica que el paciente tenga que asistir casi todos los días a los centros de salud, le incrementa los gastos de desplazamiento y la necesidad de disponer de tiempo. Desde el ámbito biológico se pueden identificar limitantes para la adherencia al tratamiento, como lo afirma un trabajador en la valoración clínica de un paciente con la enfermedad: P16: (16:28) "El señor estaba desnutrido, tenía de base al parecer un VIH, presentando otras comorbilidades, Io cual representa una mayor infección, un mayor padecimiento". Lo descrito, muestra la necesidad de tener una visión integral, y no fragmentada, en el abordaje de las diferentes comorbilidades y particularidades presentes en los sujetos de atención. También se identifica como limitante para la adherencia, desde la perspectiva de los trabajadores de la salud, la aparición de la multidrogorresistencia a los medicamentos; esta problemática tiene una repercusión colectiva, dada la posibilidad de transmisión de la enfermedad.

Ahora bien, fue mencionado por los trabajadores, la asociación VIH/SIDA y tuberculosis, En este sentido, un trabajador afirma: P4 (14:64); "es muy duro que a ti te digan que tienes tuberculosis cuando ya tienes VIH"; P4: (14:64) "O sea, no quieren vivir, no quieren, no quieren, yo tuve un paciente así, luché con él hasta el último momento, hasta que se murió. O sea, ¡Él no quería vivir! Cuando tú no quieres vivir, es muy terrible". El diagnóstico de coinfección genera un alto impacto psicosocial, que se expresa en desesperanza y negación, incluso ante la vida, lo cual limita la posibilidad de brindar un tratamiento.

\section{- Aspectos programáticos que inciden en la adherencia}

Los trabajadores de la salud reconocen que desde los aspectos programáticos existen limitantes para la adherencia al tratamiento de la tuberculosis; al respecto, un trabajador menciona: P8 (8:8) "Yo creo que radica en muchas cosas..., va desde el proceso administrativo, de los 20 minutos, de que venga por esto, la llenada de papelería... Encontrar un paciente eso implica hacer otra fórmula, implica más tiempo".

La relación paciente-trabajador de la salud se identifica desde lo programático como un factor clave que puede favorecer o limitar la adherencia al tratamiento. Dentro de los aspectos que pueden limitar la adherencia se señala el trato deshumanizado, las 
malas actitudes, la indisposición; así se alude en el siguiente enunciado: P18: (18:60) "Entonces si el médico sabe llegarle al paciente le va a ofrecer información, que le va a dar mucha tranquilidad, o si el médico no lo hace adecuadamente es un paciente que desde ese momento va a tener serios problemas, entonces seguramente no va a hacer adherencia, va a tener muchos conflictos con el tratamiento, con su enfermedad con su familia"; P7: (7:14) "En esos 10 días son críticos. La idea es cogerlo y que ya en esos dos días empiece el tratamiento"; P7: (7:14) "Si uno deja más de una semana de que el paciente vuelva acá, él se puede desanimar, no viene".

También se identificó en las entrevistas a los trabajadores de la salud que la organización de la atención puede limitar la adherencia, según se menciona a continuación: P4: (4:29) "Si un paciente viene aquí, empezando por el vigilante sabe que tiene tuberculosis y desde ahí está el rechazo, la persona no viene"; P6: (36:36) "Yo creo que, primero que todo, depende del trato que a mí me den, porque si yo llego y me tratan despectivamente, iqué jartera ir allá!". Lo anterior indica que en el proceso de atención en las instituciones de salud, reconoce que todos los funcionarios, asistenciales y administrativos, son fundamentales en la facilidad de acceso en las instituciones y claves en el proceso adherencia.

La organización de las instituciones de salud y de su red adscrita es determinante para la adherencia, en este sentido, afirma un trabajador: P7: (7:11) "Lo limita también el sitio de residencia: los pacientes que viven lejos, el niño vive en Funza, entonces, la mamá trabaja y el niño en su colegio. Es decir, lo limita eso, para no poder venir diariamente"; P8:(8:6) "Los tratamientos largos. Un paciente que tenga que ir a su EPS en horas exactas... a reclamar sus medicamentos. Es complicado, porque no siempre va a tener para el transporte". Es importante destacar que los aspectos mencionados, entre otros, son relevantes para el análisis de la organización del programa de tuberculosis y el sistema de salud.

También, los trabajadores de la salud identifican, dentro de los aspectos que pueden limitar la adherencia al tratamiento, la escases de recursos financieros y económicos para el programa de Control de Tuberculosis, P18: (18:89) "No tenemos recursos para las poblaciones vulnerables de zonas distantes, no hay recurso específico".

\section{- Relación intersubjetiva en los trabajadores de la salud/pacientes:}

\section{El trato en la atención, la formación limitada.}

El desconocimiento de la enfermedad por parte de los trabajadores de la salud es un factor que limita la adherencia, como se afirma en el siguiente texto: P16: (16:16) "el personal de salud no es muy accesible a entrar a la habitación. Solamente la jefe ingresa, obviamente, con el tapabocas adecuado, pero todavía se tiene la creencia del contagio en el contacto directo, pues a pesar de que el paciente tenga tratamiento, el contagio se va a dar".

\section{Formación limitada centrada en lo biológico.}

En relación con la categoría tuberculosis-formación académica, un trabajador de la salud menciona en su discurso la limitante que representa esta situación: P8: (42:42) "Yo creo que hay una debilidad de las universidades en este momento, es que en todas las áreas de salud se basa el 80 o 90\% en clínicas especificas y el 10\% es salud pública". Dicha formación académica limitada se reafirma en el siguiente texto: 
P8: (42:42) "tiene que ser un cambio desde la misma academia, porque los estudiantes en las universidades ven salud pública como la costura, como lo menos importante, como lo que juhhh...! Sí, jeso no es nada!... se les da un brochazo". Se identifica de esta manera la necesidad de orientar la formación académica de los trabajadores de la salud en los aspectos clínicos y de salud pública de manera integrada; estas acciones deben ser transversales y presentes en todos los escenarios de intervenciones en los que los trabajadores de la salud se desempeñan.

Precisando esta situación, un trabajador de la salud menciona: P9: (44:44) "Durante la formación, lo que hace uno es qué son los pacientes respiratorios, por ejemplo, cuáles son las medidas de aislamiento y cuáles son las patologías que lo ameritan". La situación descrita por el trabajador de la salud indica una formación limitada, que no permite comprender el proceso salud-enfermedad en su totalidad y en la complejidad que representa en el ser humano.

Así es mencionado por uno de los trabajadores de la salud, quien relata una experiencia durante su primera visita domiciliaria a un paciente: P10: (17:17) "Voy a pasar por la pena de la ignorancia de preguntarle a mi compañera: Bueno, ¿tú no te vas a poner nada? ¿No trajiste tapabocas, guantes, nada? Ahí fue cuando la enfermera entró a explicarme todo...Ya ha pasado el tiempo, dependiendo del caso, a los 15 días el paciente ya tuvo que haber iniciado obligatoriamente su tratamiento, entonces, con 15 días ya no se da la transmisión”. No conocer la enfermedad limita la posibilidad de desarrollar acciones objetivas y centradas acordes con los direccionamientos en salud y la realidad del paciente.

\section{Condiciones de trabajo y la exposición laboral en los trabajadores de la salud}

Dentro de la categoría trabajadores de la salud se identifica la exposición laboral de estos ante la tuberculosis, teniendo en cuenta su frecuente exposición como resultado del proceso de trabajo en la atención a pacientes con diferentes sintomatologías respiratorias. El trabajador de la salud manifiesta sentir temor ante el contagio: P12: (17:17) "A no, eso sí, todavía las personas le tienen como mucho miedo (risas) a la tuberculosis y a ser contagiado, pero siempre usamos medidas extras (risas) que son medidas de aislamiento respiratorio". El trabajador de la salud reconoce que está expuesto a la tuberculosis, e identifica como necesario utilizar medidas de protección personal.

El desconocimiento de la situación de exposición laboral a la tuberculosis es una situación compleja que involucra diferentes actores, que identifica el trabajador en el siguiente texto: P18 (5:23) "y el trabajador de la salud está en la mitad con una prueba de tuberculina que no sabe qué hacer con una prueba, positiva... negativa, que le genera angustia, que no sabe desde hace cuánto, no sabe qué hacer". Finalmente, otro trabajador de la salud menciona: P18 (5:23) "yo creo que la tuberculosis tiende a transmitirse muchísimo más fácilmente, y en general no estamos preparados para entender lo que pasa. Recientemente aplicamos PPD a trabajadores de tercer nivel, $y$ a la enfermera que lideró el proceso están que la linchan, porque nadie entiende qué es la tuberculosis; ni las Aseguradoras de Riesgos Profesionales están muy conscientes de cuál es su papel; entonces las se la mandan a las EPS, entonces todo el mundo se tira la pelota". 
Lo anterior muestra que la enfermedad requiere estudios que permitan describir la situación de exposición y reconozcan la enfermedad de origen profesional en los trabajadores del sector salud.

\section{Factores que fortalecen la adherencia al tratamiento}

Los aspectos cognitivos, comportamentales, sociales y programáticos y la relación paciente-trabajador de la salud son identificados por los trabajadores de la salud como factores que fortalecen la adherencia del tratamiento de la tuberculosis (ver gráfico N. $\left.{ }^{\circ} 3\right)$.

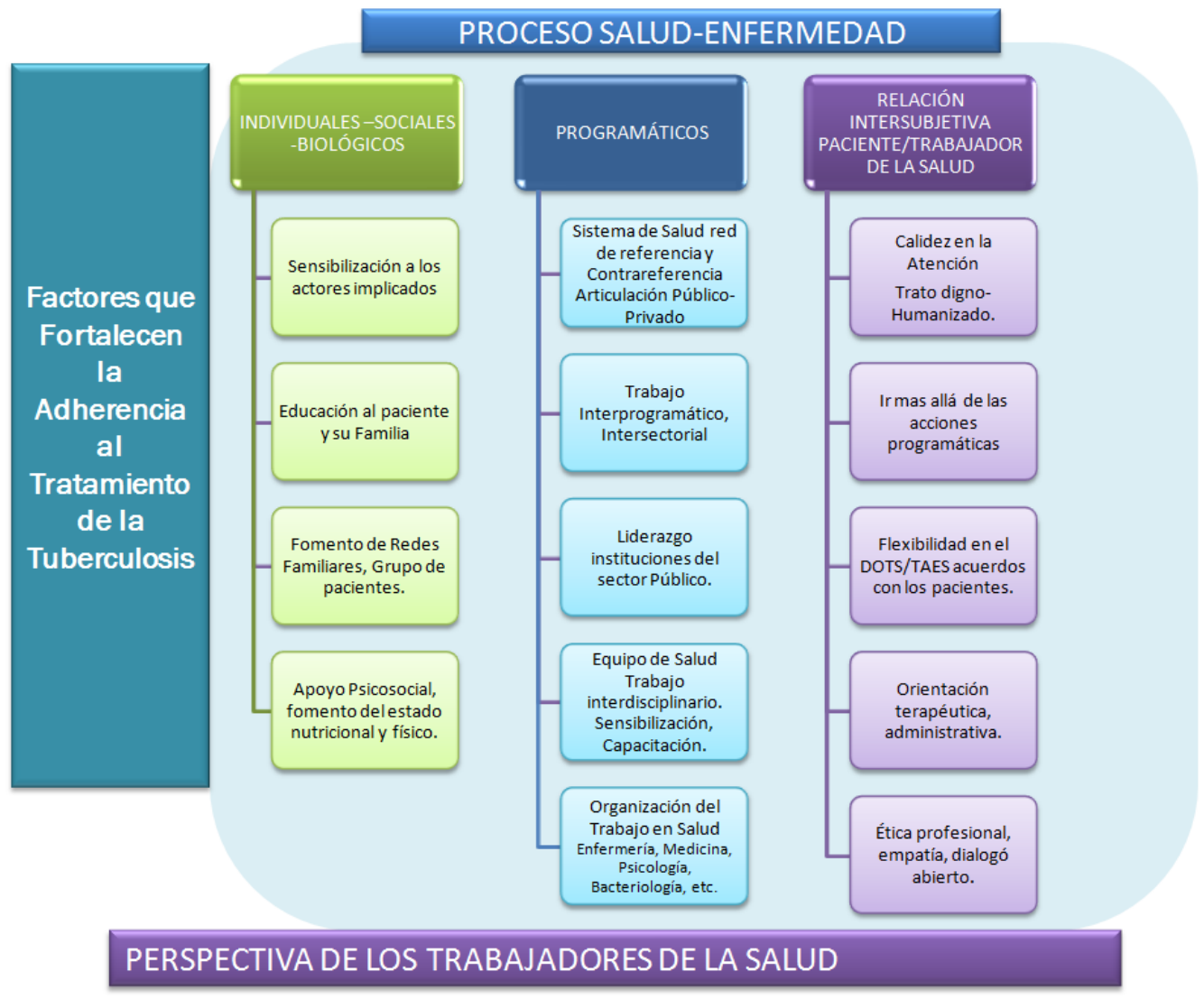

Gráfico No3. Factores que fortalecen la adherencia al tratamiento de la tuberculosis en los pacientes de la localidad estudiada, Bogotá, 2010

Como ha sido mencionado en diferentes ocasiones, debe considerarse que la tuberculosis representa una particularidad y subjetividad en las acciones en salud. Desde la perspectiva de los trabajadores de la salud, en un primer componente se destaca la necesidad de sensibilizar a los pacientes para desmitificar creencias y estigmas en torno a la enfermedad; así afirma un trabajador de la salud: P11: (11:64) "La asesoría constante también, la educación, la información, y para él. ¿Para qué? Para que él pueda entender su enfermedad, desde un comienzo".

Estas acciones son de gran importancia y deben ser impartidas por personal entrenado, que en el primer momento de consulta pueda brindar un asesoramiento terapéutico que le permita al paciente comprender su situación e identificar el tratamiento como la solución. El trabajador de la salud, en el proceso de atención al paciente con tuberculosis, debe proporcionar en la consulta tranquilidad, teniendo en 
cuenta que la persona atraviesa por un momento de mucha receptividad. Así afirma un trabajador: P11: (11:65) "desde que llegué le empecé a decir: su diagnóstico es tal, no se preocupe. Mire, hay un proceso, un tratamiento. El tratamiento va a durar tanto, es curable, usted no se va a morir".

La situación descrita facilita la adaptación de los pacientes para la toma del tratamiento, la desmitificación de falsas creencias y el fortalecimiento del proceso de adherencia. Enseguida, en este proceso de consulta al paciente con la enfermedad, debe generarse un diálogo que permita la comunicación efectiva, que se le permita al paciente hablar, comunicar sus temores, sus miedos. Desde la perspectiva del trabajador de la salud, se debe ser convincente con la orientación que suministre: P18: (4:12) "si el médico le explica, le hace ver la importancia de que se tome el tratamiento completo, de que lo va a curar y de que si no se lo toma se va a morir, entonces el médico debe ser muy convincente y explicarle al paciente". La información que brindan los trabajadores de la salud juega un papel fundamental y, en este sentido, necesita otros elementos, como los conocimientos, su experiencia en el programa, el entrenamiento que ha recibido para la formulación del tratamiento.

Así afirma un trabajador: P12 (12:38) "las visitas a los pacientes les ayuda a afrontar el cambio en su situación salud enfermedad". En este sentido, fomentar la interacción social, sumada al componente educativo, al tratamiento, brinda al individuo y su familia elementos que les permiten retornar a sus actividades cotidianas y la restitución de la dinámica social, los cual fortalece la adherencia.

Dentro de esta categoría también se considera importante la sensibilización y capacitación a los mismos trabajadores de la salud, como forma de fortalecer la adherencia. De este modo, se plantea la siguiente situación por parte de un trabajador: P18: (3:45) "hay gente que ha llegado a nuestras capacitaciones muy ruda, muy como que no les interesa, como que menosprecian un poco, y al final hasta lloran y expresan al final de las capacitaciones todo el impacto que ha tenido el hecho de entender qué es la tuberculosis, y todos los errores que ellos han cometido en el pasado los remueve"; P10: (17:17) "Luego de que ella me explicó, ahí uno va viendo ya como que se fueron quitando los miedos, despejando las dudas, quitando el estigma".

Entender el fenómeno social y biológico de la tuberculosis por parte de los actores implicados en la atención en salud dinamiza las acciones programáticas, el trato digno, la humanización en los servicios de salud. En este orden de ideas, las capacitaciones en tuberculosis y las estrategias de sensibilización deben ser próximas a las vivencias de la realidad de las personas y contribuyen al fomento de la adherencia; P18: (3:45) "Porque en los talleres que nosotros hacemos tratamos de sensibilizarlos muchísimo de que ese ser humano que está frente a él tiene dolor, tiene angustia”.

El tratamiento de la tuberculosis desde el nivel programático implica la organización de la red de referencia y contrarreferencia, la cual involucra acciones administrativas para disponer del tratamiento: P16: (37:42) "yo lo solicito y la IPS lo asume. Nos apoyamos en la red pública por lo que ellos, obviamente, tienen programa. iTienen que tener programa! Y no, el Hospital de Fontibón, súper, súper. Todo lo que hemos necesitados de todos los programas, nos han ayudado". En este sentido, se identifica la organización de la red de atención, su liderazgo desde el programa de las instituciones de salud públicas. Dentro de las directrices del programa se espera que 
los actores institucionales, tanto públicos como privados, involucrados en la atención en salud generen los mecanismos para garantizar a sus afiliados un diagnóstico y tratamiento efectivos.

En el escenario local del objeto de estudio, se identifica por los trabajadores de la salud aspectos interprogramáticos en el programa de Control de Tuberculosis de la ciudad de Bogotá; así lo menciona un trabajador: P18: (36:36) "El proyecto de Transmisibles nos permite la coordinación con un ente que se llama la Secretaría de Integración Social, para brindarles esas opciones que ellos no tenían de alimento, de vivienda, de educación, de cuidado, entonces, al tener ese soporte, el paciente se adhiere al tratamiento, $y$ es allí cuando tenemos testimonios maravillosos". E trabajador identifica la existencia de un programa formal para el control de la tuberculosis dentro de las llamadas enfermedades transmisibles y su articulación con otros programas en ámbitos sociales en las acciones de salud.

Se identifica en este sentido, por otro trabajador de la salud, el enfoque desarrollado en el programa local y distrital: P18 (47:47) "se desarrolla la secretaría de salud con el ámbito de los escolares, ámbito laboral, con el ámbito familiar, es decir, con todos los demás procesos que se dan en la secretaría hay una conexión permanente, entonces ellos nos remiten pacientes o nos ayudan, por ejemplo, a la administración de los tratamientos". Las acciones interprogramáticas del programa de tuberculosis, al articular diferentes ámbitos, tienen un impacto en diferentes grupos poblacionales, lo cual amplía el área de acción y conforma una red en el programa de Control de Tuberculosis. El trabajador de la salud señala: P18: (47:47) "Porque por ejemplo hay gente muy pobre, que vive muy lejos y que tiene dificultades por discapacidad visual, física, y que no se pueden trasladar, entonces el medicamento se lo administra un auxiliar que va hasta su casa y le brinda el tratamiento".

Finalmente, desde los aspectos programáticos que fortalecen la adherencia se identifican los actores involucrados en la atención en salud (médicos, enfermeras, bacteriólogas y, en particular, la referente del programa local de la tuberculosis). La referente del programa local es identificada como un actor clave para los trabajadores de la salud, los pacientes y las instituciones de salud adscritas al programa de control de tuberculosis.

En resumen, el abordaje del paciente con tuberculosis y las acciones programáticas de la atención en salud trascienden el ámbito disciplinar, dada la complejidad que representa el proceso Salud-Enfermedad-Tuberculosis. Así afirma un trabajador: P2: (4:54) "claro, claro, eso es clave. Y en relación con los equipos de salud integrados puede influir en la adherencia. Mis compañeras de VIH también están con transmisibles, la psicóloga... o sea, está todo un equipo junto. No sé si en otras localidades ocurrirá los mismo, pero aquí se maneja todo junto el referente de salud mental y vamos de la mano con el paciente". Así, el trabajo en el programa de tuberculosis involucra acciones conjuntas de diferentes profesionales, de las áreas biológica, social, humanas, es decir, la concepción de un equipo interdisciplinar.

Finalmente, la calidez en la atención y la buena relación entre el trabajador de la salud y el paciente contribuyen a fortalecer la adherencia de estos al tratamiento de la tuberculosis; así se plantea en el siguiente apartado: P18: (46:46) "Definitivamente, es la calidez de la persona que atiende, si la persona que atiende no tiene ese carisma, esa capacidad de llegarle a ese ser humano que está con angustia, con dolor, va a tener problemas". 
El trato al paciente implica una actitud abierta de dialogicidad entre seres, soportada por la empatía del trabajador de la salud y en la capacidad de generar confianza y seguridad a la persona. Estos elementos recaen directamente en la adherencia, es decir, en que el paciente cumpla con la toma de su tratamiento y acuda a sus controles. La construcción de la adherencia se identifica como un fenómeno complejo, y se encuentra su esencia en la relación de los actores implicados.

\section{DISCUSIÓN}

Es importante iniciar destacando que el proceso salud-enfermedad es una categoría fundamental para el estudio y el análisis de la adherencia al tratamiento de la tuberculosis. El proceso salud-enfermedad-tuberculosis, desde los significados de los trabajadores de la salud de la localidad del estudio, se representa como un fenómeno complejo, íntimamente ligado a las condiciones de vida de la población y permeado por creencias culturales, actitudes y conocimientos que definen su percepción.

En estudio realizado por Muñoz y Bertolozzi en la ciudad de Sao Pablo, Brasil, se identificó que las percepciones de la enfermedad pueden condicionar las prácticas de cuidado a los pacientes e influir en la adherencia al tratamiento, la perpetuación del estigma y las estrategias para el control de la enfermedad ${ }^{9}$. Lo anterior destaca la importancia de conocer los significados y percepciones en los trabajadores de la salud, quienes constituyen un elemento fundamental para los sistemas de salud; en este sentido, es de carácter relevante indagar en estos aspectos desde los diversos escenarios de estudio. La comprensión del significado de tuberculosis y su proceso histórico social posibilita intervenciones centradas en el contexto de los sujetos de cuidado, pensando en que la tuberculosis persiste en el escenario epidemiológico actual, a pesar de que existe un tratamiento efectivo que garantiza su curación ${ }^{10}$.

Por otro lado, desde los significados de los trabajadores de la salud de la localidad el proceso salud-enfermedad-tuberculosis representa un panorama fuertemente ligado al estigma. El estigma o prejuicio es definido por Barreiro (2000) y González (1987), citados por De la Cruz ${ }^{11}$, como una marca o una señal negativa, que se concibe en el imaginario de la sociedad al aludir o mencionar la enfermedad. Al respecto, Souza, Denise y Guerreiro ${ }^{12}$ mencionan que la tuberculosis está dada por las creencias tradicionales sobre la naturaleza, salud moral, la enfermedad y el sufrimiento humano.

La construcción del estigma radica en gran parte en el desconocimiento, las creencias y las actitudes, definidas por Forgas Berdet como "las ideas y convicciones que tenemos por verdaderas, tanto las que son permisivas como las limitantes, y que son la base de nuestra acción diaria. Son afirmaciones o negaciones sobre nuestra interpretación del mundo y sobre nuestra propia persona, sus posibilidades y limitaciones" 13 .

Jaramillo menciona que el paciente con tuberculosis, generalmente, va a ocultar su situación frente a sus familiares, amigos, la sociedad, lo cual origina sentimientos de rechazo, de temor que tienen como base el prejuicio y el estigma ${ }^{14}$. Esta situación también fue expresada por los trabajadores de la salud del presente estudio, quienes afirman que en muchas ocasiones el estigma desencadena que los pacientes opten por el camino del no tratamiento o su abandono, lo cual trae consigo complicaciones de la enfermedad como la multidrogorresistencia o incluso la muerte. El estigma a la enfermedad tiene un nexo desde la sociedad y se fortalece por el desconocimiento, las falsas creencias, los mitos, que se reproducen de generación en generación y que 
alejan la comprensión de la realidad y que convierten una limitante para su diagnóstico y control.

Es real, entonces, que existen estigmas desde la construcción social de la enfermedad, e incluso llama la atención que el estigma puede ser dado desde los mismos trabajadores de la salud. Los sujetos de estudio reconocen que no conocer la enfermedad aumenta su temor durante la atención, bien sea por desconocimiento, por creencias o formación limitada. El estigma se evidencia en el rechazo al portador, en su discriminación que afecta la comprensión de su proceso salud-enfermedad. La situación de estigma, según los trabajadores de la salud puede generarse desde el mismo paciente, su familia y los mismos trabajadores de la salud; al respecto, se menciona la siguiente situación: P4: (4:26) "Es muy difícil porque los propios trabajadores están con el estigma de la tuberculosis. Las peleas que yo tengo, el día a día en el hospital porque el paciente con tuberculosis me lo tienen aisladísimo".

En esta perspectiva, los profesionales de salud deben desempeñar un papel protagónico en acciones desde el orden político, económico, de salud y que se reflejen en el escenario local, nacional e internacional mediante la investigación y la divulgación de resultados que impacten de manera certera en los imaginarios y prejuicios sociales. La tuberculosis debe ser ampliamente discutida y ser prioridad desde todos los aspectos que confluyan en el cumplimiento de los objetivos del milenio, entre ellos el fortalecimiento de la adherencia.

Específicamente, en relación a la adherencia al tratamiento, el diccionario de la Real Academia Española señala que el término adherencia proviene del latín adhaerentia y significa "1. Unión física, pegadura de las cosas. 2. Cualidad de adherente; 3. Enlace, conexión, parentesco" ${ }^{15}$. En la revisión de literatura se encontró que Maher et ál. invitan a usar, en lugar del término adherencia, el término en inglés concordance, que “... indica que el paciente esté de acuerdo en su tratamiento, llegando así la adherencia" ${ }^{16}$; concordance, según estos autores, implica el acuerdo entre el usuario y el trabajador de la salud, con relación a cuándo los medicamentos serán tomados, siempre preservando los derechos civiles y de salud. La OMS ${ }^{17}$ advierte que la adherencia terapéutica de enfermedades como la tuberculosis se debe abordar con una panorámica amplia, dinámica, como un proceso permeado de múltiples influencias.

De acuerdo con lo anterior, la adherencia a un tratamiento no se limita a la toma de un esquema de medicamentos por el paciente, incorpora también la ejecución de cambios en las actividades de la vida diaria, la cultura de autocuidado, la asistencia a las consultas médicas programadas, entre otras tareas, todas ellas directamente relacionadas con el comportamiento del individuo, teniendo en cuenta procesos individuales, sociales y culturales que circunscriben el realizar esta actividad. De igual forma, reconoce la responsabilidad del personal de salud en el proceso de atención, dado que debe permitir instaurar una relación empática con el individuo afectado y ofrecer con claridad toda la información que este pueda necesitar.

La OMS define la adherencia al tratamiento como "El grado en que el comportamiento de una persona -tomar el medicamento, seguir un régimen alimentario y ejecutar cambios del modo de vida- se corresponde con las recomendaciones acordadas de un prestador de asistencia sanitaria" 17; como se puede ver, esta definición centra el concepto en acciones a cargo exclusivo del paciente, como el seguimiento de 
instrucciones, lo que no permite entender la adherencia como un fenómeno en donde se ven involucrados varios aspectos que superan el ámbito individual.

Di Mateo y Di Nicola ${ }^{18}$, refieren que en la adherencia al tratamiento el paciente debe jugar un papel activo, es decir, que debe participar en la toma de decisiones de su tratamiento, desde su formulación, la aceptación de este con el personal médico y colaboración en su establecimiento y seguimiento, es decir, se le debe permitir al paciente hacer parte del proceso salud-enfermedad.

La definición más citada de adherencia es la de Haynes: "medida en que una persona realiza acciones en términos de tomar medicamentos, seguir las recomendaciones nutricionales y los cambios de estilo de vida de acuerdo con las orientaciones médicas" 19. También se menciona el término "compliance", traducido como "cumplimiento terapéutico", que implica el derecho de las autoridades de salud pública para la demanda de adhesión o como un indicador para evaluar la adherencia; por ejemplo, si los pacientes se adhieren el $95 \%$ de las veces a la medicación de tuberculosis como se prescribe, se dice que su compliance es alta, mientras que pacientes con tasas bajas de adherencia tienen su compliance baja. Se destaca la necesidad, en el caso de tratamiento antituberculosis, de educar y entrenar el equipo de salud sobre acciones específicas en los programas de control, como realizar consejerías previas al inicio del tratamiento, haciendo énfasis en los efectos adversos que se pueden presentar y sus indicaciones, ya que se ha demostrado en estudios la relación entre estos y el abandono ${ }^{19}$.

El fenómeno de adherencia exige el monitoreo continuo del paciente durante el tratamiento, por ejemplo, en la revisión sistemática y organizada de los registros del programa, su asistencia a citas de seguimiento y su demanda de medicamentos. Este monitoreo se puede medir al final de las intervenciones planteadas por medio de indicadores como la tasa de curación de pacientes, resultados que pueden indicar la efectividad de las acciones programáticas contempladas en el DOTS (Directly Observed Therapy Short Course) o TAES (Tratamiento Acortado Estrictamente Supervisado) ${ }^{20}$.

Un estudio realizado en Sao Paulo verificó que el DOTS es una fortaleza para la adherencia al tratamiento, pues contribuye a ella de forma positiva, según los trabajadores de la salud, porque permite mayor contacto y acompañamiento del paciente, la toma correcta de medicamentos e identifica las necesidades de los pacientes ${ }^{21}$

En el presente estudio, estos hallazgos fueron corroborados, no obstante, se hace necesario revisar y trascender el análisis de indicadores clásicos, incluyendo otros que midan la adherencia en sus reales dimensiones, por ejemplo, soporte efectuado por otras áreas, profesionales, reuniones con redes de apoyo, acompañamiento familiar, etc., dadas las particularidades del contexto colombiano.

La no adherencia al tratamiento es uno de los mayores obstáculos para el control de la tuberculosis; ocasiona altos costos a los sistemas de salud, pérdidas de años de vida en personas jóvenes y aumento de la posibilidad de transmisión del bacilo y de la resistencia a los fármacos, entre otros. La multidrogorresistencia es una de las mayores amenazas que enfrenta el control de la tuberculosis; las fallas en el uso de los fármacos, desde su inicio, representan la mayor la posibilidad de desarrollo de resistencia a estos ${ }^{22}$. 
Cáceres y Orozco ${ }^{23}$, en un estudio de cohortes de pacientes adultos con tuberculosis, residentes en área urbana, para determinar la incidencia y los factores asociados al abandono del tratamiento antituberculoso, y el desconocer la enfermedad. Sin embargo los trabajadores de la salud participantes del presente estudio la reconocen en su permanencia y persistencia. Un trabajador menciona lo siguiente: P4 (4: 28) "EI cuento de que es reemergente, para mí, no es reemergente: ella siempre ha estado aquí y está con nosotros mientras vivimos con la situación socioeconómica que tenemos". Lo expresado por el trabajador reitera que la enfermedad no está erradicada, ni ha desaparecido, como comúnmente en el imaginario social se piensa, y que hoy por hoy, como en épocas anteriores, amerita un análisis profundo de los determinantes causales en la construcción del proceso salud-enfermedad (PSE), como las condiciones de vida, entre otros aspectos.

Por otro lado, como factores limitantes para la adherencia se encontraron la demora en el diagnóstico, la condición socioeconómica limitada, haber estar detenido, tener más de dos faltas al tratamiento y ser portador de VIH/SIDA. Cáceres y Orozco alertan sobre la necesidad de desarrollar continuamente acciones de promoción de la salud y prevención de la enfermedad, que permitan, una vez detectado un afectado, hacerle seguimiento ${ }^{23 .}$

El cumplimiento del tratamiento contra la tuberculosis se convierte en un indicador que se debe monitorear de forma permanente, para evitar la no adherencia, problemática complicada que contribuye a la multidrogorresistencia, al aumento de la mortalidad de los pacientes y a la perpetuación de la cadena de transmisión de la enfermedad. Llama la atención que los datos de Colombia para el 2010 son parciales en el informe global de tuberculosis de la OMS, lo que muestra dificultades en la información, asunto que puede afectar la posterior toma de decisiones.

Las fallas en los programas de control de la tuberculosis están relacionadas con causas más estructurales. Estudios realizados en tres municipios de Colombia analizaron las consecuencias de la reforma de salud en el país para los programas de salud pública a nivel local, entre ellos el programa de control de tuberculosis, y concluyeron que las reformas del Sistema de Salud afectan la operacionalización de los programas de salud pública ${ }^{24}$.

Un estudio ${ }^{25}$ realizado en Buenaventura (Valle) entre los años 1997 y 2001 encontró una elevada resistencia inicial a medicamentos antituberculoso de primera línea en personas con diagnóstico de la enfermedad; la resistencia inicial a cualquier medicamento fue de $25 \%(9 / 36)$ y $32 \%(23 / 72)$, en la primera y segunda encuesta, respectivamente; la multirrestencia inicial (resistencia, al menos, a isoniacida y rifampicina) fue de $6 \%$ en ambas encuestas. Estos hallazgos alertan sobre la necesidad de la detección precoz y de realizar acciones de vigilancia epidemiológica que eviten la resistencia, toda vez que se convierte en una barrera potente a la adherencia al tratamiento.

De esta manera, se identifica cómo la adherencia a la tuberculosis reúne procesos cognitivos y comportamentales de los trabajadores de la salud, de los pacientes, de la comunidad, y afecta el desarrollo del programa. En Costa Rica, en el año 2005, el Departamento de Fármaco-Epidemiología desarrolló una Guía de criterios técnicos basados en la evidencia para el tratamiento de la tuberculosis; sus autores anotan como factores que contribuyen a la adherencia el fácil acceso a los medicamentos, la atención sin barreras económicas, el manejo adecuado del caso, el uso de estrategias 
que recuerden el tratamiento y los controles, los tiempos mínimos de espera en los servicios, la educación sobre efectos adversos y la adecuada comunicación con el equipo de salud ${ }^{26}$. Estos factores también se contemplaron durante el desarrollo de las entrevistas del presente estudio, pero se considera de enorme importancia la amplitud y el detalle que mostraron los trabajadores de la salud.

En estudio realizado en São Paulo por Bertolozzi ${ }^{27}$ sobre adherencia al tratamiento de la tuberculosis se encontró que es concebida como un proceso complejo, que integra las dimensiones singular, particular y general de la realidad; de acuerdo con la autora, la adherencia al tratamiento de la tuberculosis está esencialmente asociada al entendimiento que el paciente tiene de su enfermedad y al lugar que ocupa en la sociedad, es decir, está articulada a las condiciones concretas de vida y de trabajo; de estas depende que los portadores de la enfermedad pueden enfrentarla o no y controlarla. Para Bertolozzi, la adherencia incluye aspectos relacionados con la organización de los servicios de salud, dentro los cuales, sobretodo, está el trato humanizado con empatía, en el cual se puedan acoger las necesidades de los pacientes por parte del los trabajadores de la salud ${ }^{27}$.

En estudio ${ }^{28}$ multicéntrico realizado en 53 hospitales de España, en los años 2006 y 2007, con 1.490 historias clínicas de pacientes, se encontró que los factores asociados a la pobre adherencia fueron ser hombre, inmigrante, paciente joven, vivir solo, paciente con coinfección $\mathrm{VIH}$, vivir confinado en instituciones, haber tenido tratamiento anterior de tuberculosis, ser usuario de drogas intravenosas y no conocer ni comprender la enfermedad: Como se puede observar, a pesar de haberse realizado en un contexto diferente al colombiano, este estudio identifica aspectos que limitan la adherencia al tratamiento que también fueron identificados desde el punto de vista de algunos de los trabajadores que participaron en el presente estudio, como la comorbilidad con VIH y el desconocimiento sobre la enfermedad; así mismo, se puede ver que existen diferentes formas de conceptuarse el abandono y el fracaso del tratamiento, comparado con las directrices colombianas.

Xu et ál., en el año 2006, en 13 distritos de Jiangsu, provincia de China, desarrollaron un estudio, con metodología mixta, para analizar el proceso de adherencia; a través del cuestionario se identificaron 670 pacientes, de los cuales se entrevistaron 20, y 10 trabajadores de la salud. El tratamiento de los datos fue a través del análisis de contenido. Se encontró que el $12,2 \%$ de los pacientes presentaron no adherencia, lo cual se asoció a factores como estar divorciado, falta de aseguramiento en los servicios de salud y ser inmigrante; otros factores asociados a no adherencia fueron: reacciones adversas a los medicamentos y estigma social. Fue menor el riesgo de no adherencia en aquellos pacientes que reinician su tratamiento. Para el caso de los pacientes en la modalidad de Tratamiento Directamente Observado y que adicionalmente recibían visitas regulares de trabajadores de la salud, el riesgo de no adherencia fue menor ${ }^{29}$.

Así, se constata en la revisión de literatura y en el presente estudio que la no adherencia al tratamiento está asociada a barreras sociales, culturales y demográficas, así como a aquellas relacionadas con el proceso de producción de servicios de salud y los problemas con los medicamentos ${ }^{30}$. Es importante destacar que el estudio aislado de estos factores puede contribuir a fortalecer el estigma de la tuberculosis, de allí la necesidad de realizar estudios que permitan ver la totalidad de 
los aspectos implicados en la adherencia de la tuberculosis, en un contexto histórico social específico.

Otro tópico ampliamente destacado en las investigaciones es el rol que desempeña el trabajador de la salud en la adherencia al tratamiento de tuberculosis, el cual es planteado ampliamente por diversos estudios ${ }^{31}$; en estos se retoma que el vínculo que se crea entre el profesional de salud y el paciente es determinante en el seguimiento del tratamiento, en su abandono o culminación, dado que su función dentro del proceso no solo se debe encaminar a verificar la toma del medicamento por medio de estrategias como el tratamiento supervisado perteneciente al DOTS, sino también incluir espacios de escucha, orientación y entendimiento con el paciente. Se espera que estas acciones posibiliten la aproximación de la concepción del proceso salud-enfermedad, las condiciones sociales y afectivas de las personas portadoras. El establecimiento de una relación entre el trabajador de la salud y el paciente permite ir más allá de la concepción biológica de la tuberculosis ${ }^{32}$ y encamina a la concepción social de ella, tan importante y definitiva en el seguimiento y probabilidades de cura de la enfermedad.

Los trabajadores reconocen la necesidad de generar estrategias novedosas en salud que contribuyan a dar un soporte social e integral al proceso de adherencia al tratamiento; estrategias que involucren diversos actores, sectores e instituciones. Así se verifica, de acuerdo con lo expresado por los trabajadores, que el concepto de adherencia también involucra atributos relacionados con el programa de control de tuberculosis, con la interacción del paciente y el trabajador de la salud, con aspectos programáticos (que, a su vez, están determinados por el sistema de salud colombiano y por las particularidades del programa en cada contexto) y con las necesidades específicas de sus usuarios.

Es necesario destacar que durante la realización del presente estudio se corroboró que en el proceso de atención de los programas de control de tuberculosis la interacción oportuna y empática del profesional de salud y del paciente constituye la mayor fortaleza para alcanzar la adherencia. Cabe aclarar que estos encuentros entre sujetos trascienden el ámbito profesional y exigen la manifestación de subjetividades de las personas implicadas en el proceso de atención. En este proceso de interacción es posible que se identifiquen de forma oportuna fortalezas y limitantes para alcanzar la adherencia al tratamiento de la tuberculosis.

Este hallazgo en el escenario y con los sujetos participantes destaca la necesidad de revisar los procesos de formación de los profesionales de la salud y la organización de los servicios y programas de salud; reconoce que las acciones en salud deben revisarse de forma crítica, y destaca la profunda fortaleza cuando el profesional se involucra en el cuidado integral del paciente. Conforme apuntan Muñoz y Bertolozzi ${ }^{32}$, "el proceso de atención debe estar siempre mediado por el diálogo abierto que integre afectos, que parta de la realidad social del enfermo, de la forma como se vive y enfrenta el proceso de enfermar y la forma como se lleva la vida en lo cotidiano".

Finalmente, la adherencia al conformar un fenómeno multidimensional implica intervenciones centradas en los sujetos, que posibiliten obtener la curación de la enfermedad en los pacientes, y la prevención de la resistencia a los fármacos, grave problemática de marcada trascendencia en la salud pública. La adherencia en su trasfondo social en tanto, implica un abordaje programático que articule la intersectorialdad, la intersubjetividad la investigación, formación, y el vínculo del 
paciente y el trabajador de la salud, en su quehacer profesional, acercando el contexto social, el cambio de la percepción de la enfermedad, que permitan oportunidad para el mejoramiento de las condiciones de vida en la población y el soporte social para la conformación de la adherencia.

\section{REFERENCIAS BIBLOGRÁFICAS}

1 World Health Organization-WHO. Global Tuberculosis Control: Surveillance, planning, financing. WHO Report. 2011. ISBN 9789241564380 Disponible en http://www.who.int/tb/publications/global report/2011/gtbr11 full.pdf

2 Ministerio de la Protección Social- MPS. Plan estratégico Colombia libre de tuberculosis 2010-2015. Tercera Edición Bogotá. 2009; ISBN 978-958-8472-09-6 Consultado 24/07/2011. www.dssa.gov.co/index.php/documentos/doc_download/55-a1

3 Lilia Edith López Rojas. Documento técnico programa de Enfermedades Transmisibles. Secretaria Distrital de Salud de Bogotá. Año 2012.

4 Cruz O.A, Muñoz A.I, Florez, E.I. "Conocimientos sobre Tuberculosis en Trabajadores de la Salud en una localidad de Bogotá" . En: Colombia Revista: Avances En Enfermería ISSN:0121-5000 ed: v.24 fasc. 1 p.147 - 151,2011

5 Barroso, E. Mota S, Morais M, Campelo C, Barroso J, Rodríguez, J. Fatores associados aos tratamentos inadequados em grupo de portadores de tuberculose multirresistente. J pneumol [periódico na internet]. 2003 [citado 2004 jun. 10];29(6):[cerca de 8 p.]. Disponível em: http://www.scielo.br/pdf/jpneu/v29n6/v29n6a06.pdf

${ }^{6}$ Benevides S, Silva A, Botelho C. Abandono do tratamento da tuberculose pulmonar em Cuiabá-mt-Brasil. J Bras Pneumol, 2005; 31(5): 427-35.

7 Bertazone Ec, Gir E, Hayashida M. Situações vivenciadas pelos trabalhadores de enfermagem na assistência ao portador de tuberculose pulmonar. Rev Lat Am Enferm. Feb-2005; 13(3): 374-81.

${ }^{8}$ Gadamer H. Verdade e método. Petrópolis: vozes, 2006.

9 Muñoz Sánchez Al y Bertolozzi MR. Percepción de los trabajadores de salud de unidades básicas de salud de São Paulo (Brasil) sobre la tuberculosis. Av. Enferm. [online]. 2009 assistência ao portador de tuberculose pulmonar. Rev Lat Am Enferm. Feb-2005; 13(3): 374-81.

10 Organización Mundial de la Salud-OMS. Centro de Prensa, Nota descriptiva Tuberculosis. Washington; 2008.

11 De la Cruz A. Tuberculosis y afectividad. Instituto de medicina tropical "Pedro Kourí". Rev cubana Med. Trop. 2004; 56(3): 214-8.

${ }^{12}$ Souza S, Denise M, Guerreiro V. Passando pela experiência do tratamento para tuberculose. Contexto - Enferm. [serial on the Internet]. 2010 Dec [cited 2011 Jan 22]; 19(4): 636-643. Available from: http://www.scielo.br/scielo.php?script=sci_arttext\&pid=S0104-

07072010000400005\&lng=en.

${ }^{13}$ Forgas B. Asesoría filosófica y programación neuro-lingüística: El trabajo con las creencias. Ponencia para el VIII Congreso Internacional de Filosofía Práctica. Sevilla, España, 2006.

14 Jaramillo, E. Tuberculosis and Stigma: Predictors of Prejudice against People with Tuberculosis. Cali, Colombia Journal of Health Psychology, 4(1): 71-79; 1999

15 Real Academia Española. Diccionario de la lengua española [Internet]. Madrid; 2001...Disponíble en: http://www.rae.es 
${ }^{16}$ Maher D, Uplekar M, Blanc L, Raviglione M. Teatment of tuberculosis. BMJ. 2003; 327(7419): 822-3.

17 Organización Mundial de la Salud - Organización Panamericana de la Salud. Adherencia a los tratamientos a largo plazo. Pruebas para la acción. Washington D.C. (Estados Unidos): Organización Mundial de la Salud - Organización Panamericana de la Salud, 2004. p. 14. Disponible en http://www.paho.org/Spanish/AD/DPC/NC/ncadherencia.pdf

${ }^{18}$ Di Matteo R and Di Nicola D. Achieving Patient Compliance. The Psychology of the Medical Practitioner's Role, citados por ORTIZ P, Manuel y ORTIZ P, Eugenia. Psicología de la salud: Una clave para comprender el fenómeno de la adherencia terapéutica. En: Revista Médica de Chile [Online]. Vol.135, N. 5 (may., 2007); pp. 647-652.

${ }^{19}$ Haynes RB. Introduction. En: Haynes RB, Taylor DW, Sackett DL, eds. Compliance in health care Baltimore: John Hopkins University Press; 1979. p. 1-7

${ }^{20}$ Lwilla F, Schellenberg D, Masanja H, Acosta C, Galindo C, Aponte J, et ál. Evaluation of efficacy of community-based vs. institutional-based direct observed short-course treatment for the control of tuberculosis in Kilombero district, Tanzania. Trop Med Int Health. 2003; 8(3): 204-10.

${ }^{21}$ Bertolozzi, MR. Estratégia do tratamento diretamente observado (DOTS) no Brasil: potencialidades e limites na adesão ao tratamento [relatório técnico cnpq]. São Paulo; Escola de Enfermagem, Universidade de São Paulo; 2004.

${ }^{22}$ Schwartz DB, Adler A, Dasaro A, Debellis RJ, Dick R, Ferro TJ, et ál. Improving adherence with antimicrobial therapy for respiratory tract Infections: a discussion of directly observed therapy (dot) and short-course Therapies. Am j therap. 2004;11 suppl 1: s18-s21.

23 Cáceres-Manrique FM, Orozco-Avargas LC. Demora en el diagnóstico de tuberculosis pulmonar en una región de Colombia. Rev. Salud pública [serial on the internet]. 2008 feb [cited 2010 nov 27] ; 10(1): 94-104. Available from: http://www.scielosp.org/scielo.php?Script=sci_arttext\&pid=s0124-

00642008000100009\&Ing=en. Doi: 10.1590/s0124-00642008000100009.

${ }^{24}$ Ayala Cerna C, Kroeger A. La reforma del sector salud en Colombia y sus efectos en los programas de control de tuberculosis e inmunización. Cad. Saúde pública [serial on the internet]. 2002 dec [cited 2010 nov 27] ; 18(6): 1771-1781. Available from: $\quad$ http://www.scielo.br/scielo.php?Script=sci_arttext\&pid=s0102311x2002000600032\&lng=en. Doi: 10.1590/s0102-311x2002000600032

25 Moreira CA, Hernández HL, Arias NL, Castaño MC, Ferro BE, Jaramillo E. Resistencia inicial a drogas antituberculosas en Buenaventura, Colombia. Biomédica 2004; 24: 73-79.

${ }^{26}$ Departamento de Farmacoepidemiología. Caja Costarricense de Seguro Social. Criterios Técnicos y recomendaciones basadas en evidencia para la construcción de Guías de Práctica Clínica para el Primer y Segundo Nivel de Atención. Costa Rica; 2005.

27 Bertolozzi MR. A Adesão ao programa de controle da tuberculose no distrito Sanitário do Butantã, São Paulo [tese]. São Paulo: Faculdade de Saúde Pública, Universidade de São Paulo; 1998.

${ }^{28}$ Respira-Fundación Española Del Pulmón-Separ. Características diferenciales de la tuberculosis en inmigrantes residentes en España. Libro del año Separ-2008 sobre la Tuberculosis y la solidaridad ISBN: 78-84-936373-7-8 y D.L.: B-24125/09, consultado internet

http://www.separ.es/doc/publicaciones/libros/Libro_SEPAR_4_09_09.pdf. 
${ }^{29}$ Cayla Xu W, Lu W, Zhou Y, Zhu I, Shen H, Wang J. Adherence to anti-tuberculosis treatment among pulmonary tuberculosis patients: a qualitative and quantitative study. Bmc health services research [serial on the internet]. (2009), [cited november 17, 2010]; 9169. Available from: medline with full text.

${ }^{30}$ Mishra P. Adherence to tuberculosis treatment under directly observed treatment, short-Course (DOTS) in Nepal: quantitative and qualitative studies [thesis]. Danish: The Danish University of Pharmaceutical Sciences; 2006.

31 Arcêncio RA. A organização do tratamento supervisionado nos municípios prioritários do estado de São Paulo [dissertação]. Ribeirão Preto: Escola de Enfermagem de Ribeirão Preto, Universidade de São Paulo; 2006.

32 Muñoz Sánchez, Al y Bertolozzi MR. Más allá del DOTS (directly observed treatment short-course) en el control de la tuberculosis: medio que promueve la comunicación y la identificación de las necesidades. Revista Latinoamericana de Enfermería [online], (2009); 17(5): 689-694. 